\title{
Characterization of an Ihx1a transgenic reporter in zebrafish
}

\author{
LISA M. SWANHART1, NOBUHIRO TAKAHASHI ${ }^{2}$, RACHEL L. JACKSON ${ }^{1}$, GREGORY A. GIBSON ${ }^{3}$, \\ SIMON C. WATKINS ${ }^{3}$, IGOR B. DAWID ${ }^{2}$ and NEIL A. HUKRIEDE*,1,4 \\ ${ }^{1}$ Department of Microbiology and Molecular Genetics, University of Pittsburgh, Pittsburgh, Pennsylvania, \\ ${ }^{2}$ Laboratory of Molecular Genetics, National Institute of Child Health and Human Development, National \\ Institutes of Health, Bethesda, Maryland, ${ }^{3}$ Department of Cell Biology and Physiology, Center for Biological \\ Imaging, University of Pittsburgh, Pittsburgh, Pennsylvania, ${ }^{4}$ University of Pittsburgh Drug Discovery \\ Institute, University of Pittsburgh, Pittsburgh, PA, USA.
}

\begin{abstract}
The LIM-domain containing transcription factor, Lhx1, is involved in the regulation of early gastrulation cell movements, kidney organogenesis and other processes in vertebrate model organisms. To follow the expression of this gene in live embryos, we created transgenic zebrafish expressing enhanced green fluorescent protein (EGFP) under the control of Ihx1a regulatory regions. $T g$ (Ihx1a:EGFP) ${ }^{p t 303}$ recapitulates the expression of endogenous $I h x 1 a$ beginning at early gastrula stages through 72 hours of development with only few exceptions. In addition, over-expression of the Nodal ligand, ndr1, results in the concomitant expansion of the transgene and endogenous $/ h \times 1 a$ expression. Treatment of $T g(I h x 1 a: E G F P)^{p t 303}$ embryos with the small molecule SB-431542, an inhibitor of Nodal signaling, results in the loss of both transgene and endogenous $/ h \times 1 a$ expression. These experiments suggest that $\operatorname{Tg}(\operatorname{lhx} 1 \mathrm{a}: E G F P)^{p t 303}$ is regulated in a manner similar to endogenous Ihx1a. Therefore, this reporter can be utilized not only for monitoring Ihx1a expression, but also for numerous applications, including chemical genetics screening.
\end{abstract}

KEY WORDS: lhx1a, transgene, zebrafish, kidney, gastrulation

\section{Introduction}

LIM domain-containing proteins have been implicated in numerous biological processes including cell-fate determination, tissue-specific gene expression, neuronal pathfinding, and actin organization. This motif was originally identified within LIMhomeodomain proteins (LHX), a family of proteins of which Lhx1 (formerly known as Lim1) is a member (Dawid et al. 1998). Lhx1 plays a number of roles during the course of vertebrate development, including an important early role in gastrulation. It is expressed in the organizer region (and its functional equivalents) in Xenopus, mice, and zebrafish (Shawlot and Behringer 1995; Taira et al. 1992; Toyama and Dawid 1997), and $L h \times 1$ deficiency in both mice and Xenopus results in the loss of all cephalic structures (Hukriede etal.2003; Kodjabachian etal.2001; Shawlot and Behringer 1995).

$L h \times 1$ also regulates the development of a number of organs, including the kidney. The vertebrate kidney develops from the intermediate mesoderm (IM), and $L h \times 1$ serves as a marker of progenitor cells in this tissue (Carroll and Vize 1999; Karavanov et al. 1996; Toyama and Dawid 1997). In the mouse it continues to be expressed in both the mesonephric and metanephric kidneys where it is required for nephron formation. Loss of $L h x 1$ in mice results in the disruption of IM differentiation and the absence of functional kidneys (Shawlot and Behringer 1995).

There are multiple pathways that regulate $L h x 1$ expression during development, including Nodal, which acts in the early embryo. Members of the Nodal signaling family are TGF- $\beta$ ligands that serve as inducers of both mesoderm and endoderm and also pattern the dorsal-ventral axis of the vertebrate embryo (Schier and Talbot 2005). Nodals signal through EGF-CFC co-receptors and type I and II Activin receptors. In zebrafish, over-expression of synthetic mouse nodal RNA leads to ectopic expression of Ihx 1a in embryos beginning at 30\% epiboly (Toyama et al. 1995). In addition, a mutation in the EGF-CFC, one-eyed pinhead (oep),

\footnotetext{
Abbreviations used in this paper: EGFP, enhanced green fluorescent protein; hpf, hours post fertilization; IM, intermediate mesoderm; Lhx1, LIMhomeobox 1; ndr1, nodal-related 1.
}

\footnotetext{
*Address correspondence to: Neil A. Hukriede. 3501 5th Ave, 5601 BST3, University of Pittsburgh, Pittsburgh, PA 15213, USA. Fax: +1-412-383-5918. e-mail: hukriede@pitt.edu
} 
results in the complete loss of /hx1a expression during gastrulation (Watanabe et al. 2002).

Because of the numerous roles that $L h x 1$ plays throughout development, we created an EGFP transgene in zebrafish to follow Ihx1a expression in vivo, and to further our understanding of its regulation during gastrula stages and pronephric development. Tg(Ihx1a:EGFP) ${ }^{2} 303$ expression recapitulates Ihx1a expression at all stages analyzed, and modulation of the Nodal signaling pathway, either by over-expression of ndr1or treatment with a small molecule inhibitor, confirms that this transgene is regulated in the same manner as the endogenous $/ h \times 1 a$ gene. Therefore, Tg(Ihx1a:EGFP)ot303 should serve as a useful resource for investigating gastrulation and mechanisms of cell-fate specification within the kidney.

\section{Results}

\section{Generation of Ihx1a transgenic line}

A BAC clone containing the Ihx1a genomic region was identified and an $8.8 \mathrm{~kb}$ fragment containing 5' untranslated sequence, exon 1, and part of exon 2 was isolated and subcloned (Fig. 1A). This fragment contains the first intron, which is important for transcriptional regulation of both the Xlim 1 and $/ h \times 1 a$ genes in Xenopus and zebrafish, respectively (Rebbert and Dawid 1997; Watanabe et al. 2002). EGFP was inserted downstream of the start codon and the Ihx1a:EGFPDNA fragment was cloned into the pl-Scel meganuclease vector for generating transgenic lines (Thermes et al. 2002). The injected embryos were raised to adulthood and screened for germline carriers by EGFP expression. Three founder transgenics were identified and while levels of expression varied between lines, the expression patterns were similar. Homozygous lines were created from these founders (allele designations: pt301, pt302, pt303). $\mathrm{Tg}$ (Ihx 1a:EGFP)fish are fecund and develop normally suggesting that the insertion of this transgene does not cause any deleterious effects. For all subsequent experiments, we used Tg(Ihx1a:EGFP)t303, the line with the strongest expression.

\section{Early expression of $\mathrm{Tg}(\mathrm{Ihx} 1 \mathrm{a}: \mathrm{EGFP})^{\mathrm{pt} 303}$}

EGFP protein was first analyzed at shield stage where it is localized to the marginal cells (Fig. $1 \mathrm{~B}, \mathrm{C}$, bracket) and the shield (Fig. 1 B,C, arrow), the fish equivalent of Spemann's Organizer in Xenopus and the mouse node. By 5-somites, EGFP protein is seen predominantly in the notochord (Fig. 1D, arrowhead) and bilaterally in the intermediate mesoderm (IM) (Fig. 1D, arrow), the

Fig. 1. Construct design and early Tg(Ihx1a:EGFP)pt303 expression. (A) Schematic of Ihx1a:EGFP construct. (B-S) Tg(Ihx1a:EGFP)pt303 embryos. (B-G) $\mathrm{Tg}\left(\mathrm{lh} \times 1 \mathrm{a}\right.$ :EGFP) ${ }^{\mathrm{pt} 303}$ expression. (H-M) In situ hybridization for egfp. (N-S) In situ hybridization for Ihx1a. (B,H,N) Shield stage embryos, animal view. $(\mathbf{C}, \mathbf{I}, \mathbf{O})$ Shield stage embryos, dorsal view. Arrow marks the shield and bracket denotes the marginal cells. (D,J,P) 5-somite stage embryos, dorsal-posterior view. Arrow points to intermediate mesoderm and arrowhead marks the notochord. $(\mathbf{E}, \mathbf{K}, \mathbf{Q})$ 5-somite stage embryos, lateral view. Anterior is to the left. (F, $\mathbf{L}, \mathbf{R}) \mathbf{1 0}$-somite stage embryos, dorsal-posterior view. Arrow points to the intermediate mesoderm and arrowhead marks the notochord. White arrow denotes anterior expression domain of $\mathrm{h} \times 1 \mathrm{a}$ in the intermediate mesoderm. $\mathbf{( G , M , S ) ~} 10$ somite stage embryos, lateral view. Anterior is to the left. Abbreviations: $D$, diencephalon; NK, neural keel; $P$, polster.

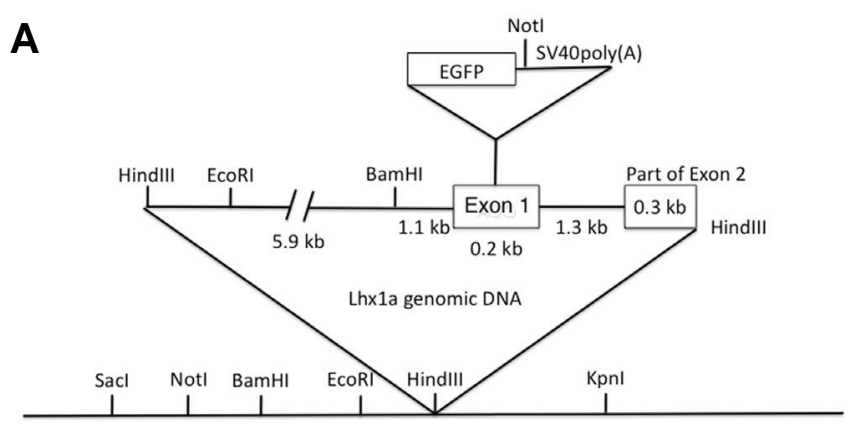

Multiple cloning site of pBluescript II KS(+)
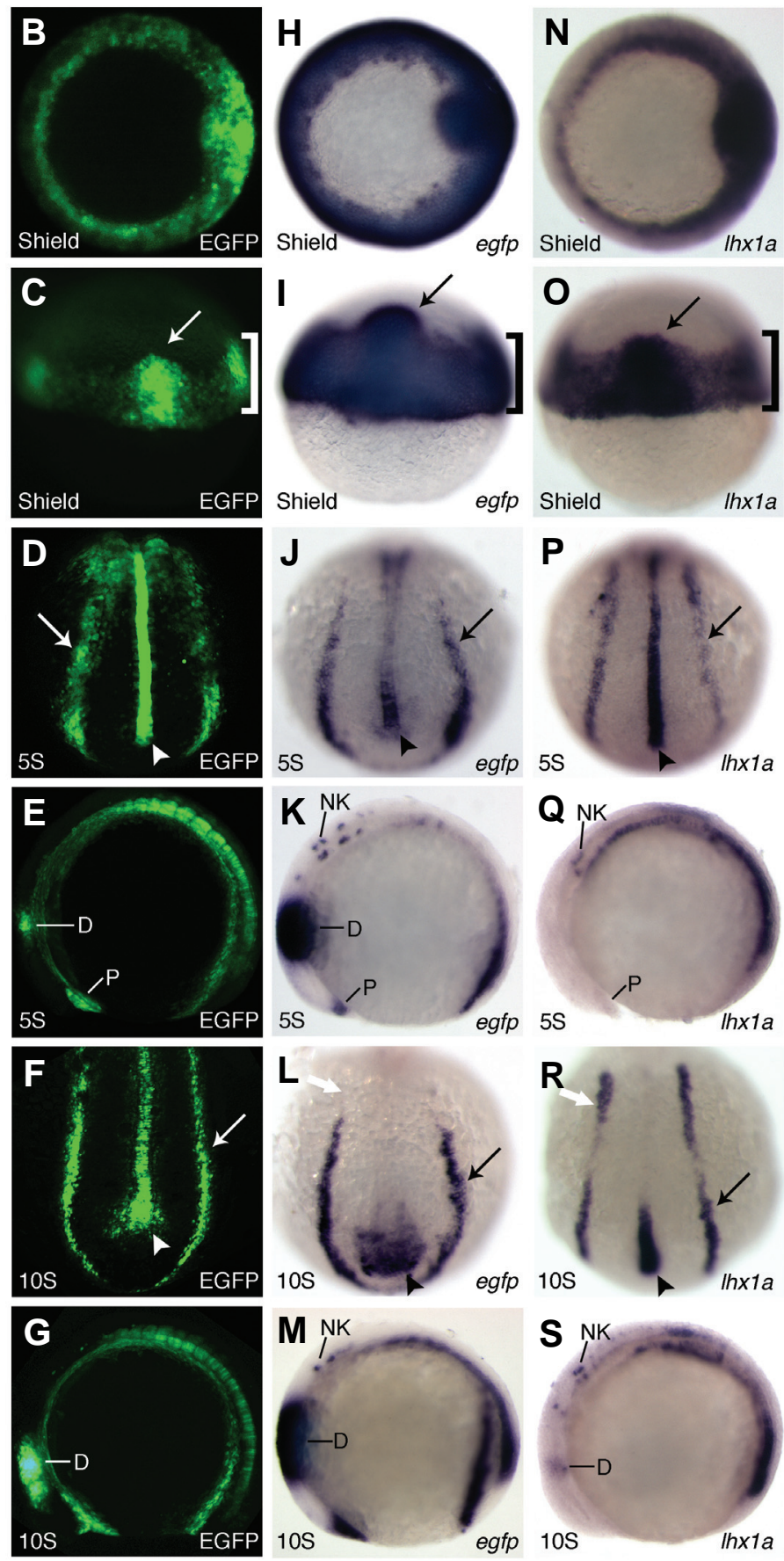
tissue that will ultimately give rise to the kidney. There is also expression in the polster and the diencephalon (Fig. 1E). By 10somites, EGFP protein remains in the most posterior region of the notochord as well as the IM (Fig. 1F, arrowhead and arrow, respectively). There is still strong expression in the diencephalon (Fig. 1G).

At the RNA level, egfp expression recapitulates what is seen with the protein, although some structures can be seen in finer detail through in situ hybridization. Similar to the protein, egfp mRNA is expressed in the marginal cells and the shield (Fig. $1 \mathrm{H}, \mathrm{I}$, bracket and arrow, respectively). At 5-somites, expression is seen throughout the notochord and the IM (Fig. 1J, arrowhead and arrow, respectively). It is present in the polster and neural keel as well as in the diencephalon where expression is aberrantly strong (Fig. 1K). By 10-somites, down-regulation of egfpexpression is beginning in the IM and the notochord (Fig. $1 \mathrm{~L}$, arrow and arrowhead, respectively). Expression is still present in the neural keel and remains strong in the diencephalon (Fig. 1M) at this stage.

The efficacy of this transgene lies in its ability to recapitulate endogenous I $\mathrm{h} \times 1 \mathrm{a}$ expression. For the majority of tissues examined, this appears to be the case. At shield stage, there is strong expression of endogenous $/ h \times 1 a$ in the shield and the margin (Fig. $1 \mathrm{~N}, \mathrm{O}$, arrow and bracket, respectively). By 5-somites, expression is predominantly seen in the notochord and IM, although weak expression can also be seen in the polster and neural keel (Fig. 1P, arrowhead and arrow, respectively and 1Q). At 10-somites, Ihx1a expression is down-regulated in the notochord and throughout most of the IM (Fig. 1R, arrowhead and arrow, respectively). While diencephalon expression is first reported at this stage, Ihx1a expression remains in the neural keel (Fig. 1S).

The anterior IM expression of Ihx1a (Fig. 1R, white arrow) ultimately contributes to proximal fates in the pronephric kidney, and presumably corresponds with the expression seen in the proximal tubule at 24 hours post fertilization (hpf) (Toyama and Dawid 1997). This expression domain is not found in the transgene (Fig. 1L, white arrow), although EGFP protein seems to perdure (Fig. 1F), suggesting variations between endogenous Ihx1a expression and Tg(Ihx1a:EGFPpt303. Overall, the expression of $T g$ (Ihx1a:EGFP)t303 agrees with the endogenous expression pattern of $/ h \times 1 a$. The main differences observed are earlier and stronger transgene expression in the diencephalon (Toyama and Dawid 1997; Toyama et al. 1995) and an absence of anterior IM expression at the 10-somite stage.

\section{$\mathrm{Tg}(\operatorname{lhx} 1 \mathrm{a}: \mathrm{EGFP})^{\mathrm{pt} 303}$ expression at 24 hpf through early larval stages}

Tg(Ihx1a:EGFP)t303 embryos at 24 hpf have a dynamic expression pattern. EGFP protein is localized to the forebrain,
Fig. 2. Expression of Tg(Ihx1a:EGFP)pt303 during later development. $A / l$ animals are oriented such that anterior is to the left. (A-P) $\operatorname{Tg}(\operatorname{lh} \times 1 \mathrm{a}: \mathrm{EGFP})^{\mathrm{pt} 303}$ embryos and larvae. $(\mathbf{A}, \mathbf{B}, \mathbf{G}, \mathbf{J}, \mathbf{M}-\mathbf{P})$ Tg(Ihx1a:EGFP)pt303 expression. (C, $\mathbf{D}, \mathbf{H}, \mathbf{K})$ In situ hybridization foregfp. $(\mathbf{E}, \mathbf{F}, \mathbf{I}, \mathbf{L})$ In situ hybridization for Ihx1a. (A-F) $24 \mathrm{hpf} \mathrm{em-}$ bryos. Arrow marks position of pronephric proximal tubule and arrowhead delineates distal tubule and duct. $(\mathbf{A}, \mathbf{C}, \mathbf{E})$ Lateral view, bracket denotes hindbrain. (B,D,F) Dorsal view. (G-I) 48 hpf larvae, arrow in $H$ marks distal tubule and duct in the kidney. (J-L) $72 \mathrm{hpf}$ larvae. (J) Montage of two images to display entire larvae. (M-P) Longitudinal optical sections of transgenic animals. (M) 24hpfembryo. (N-P) 48hpf larva. Abbreviations: $a L L$, anterior lateral line ganglia; $F B$, forebrain; $H B$, hindbrain; $M B$, midbrain; OV, otic vesicle; pLL, posterior lateral line ganglia; SC, spinal column; $T B$, tailbud.
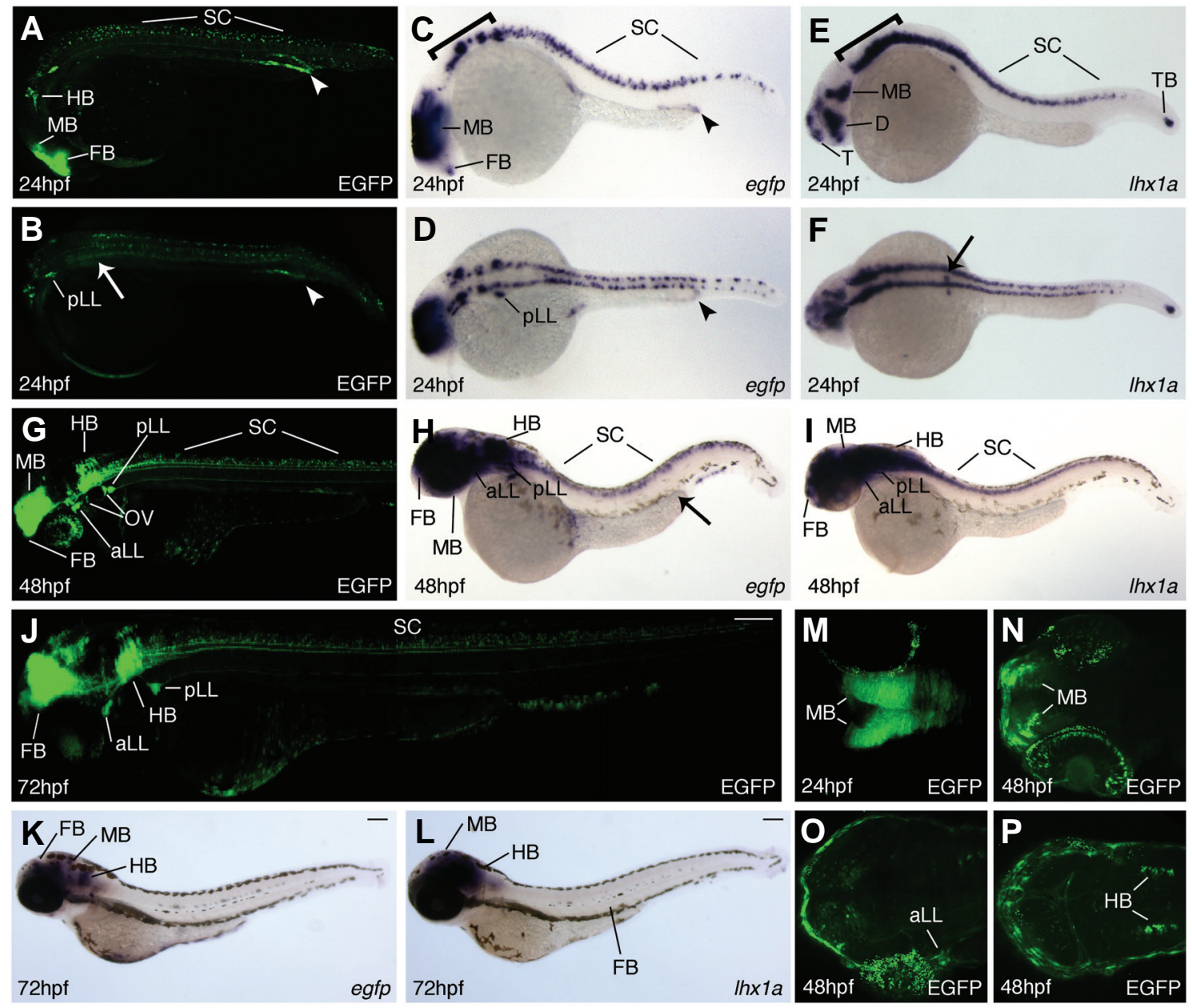

$72 \mathrm{hpf}$

egfp
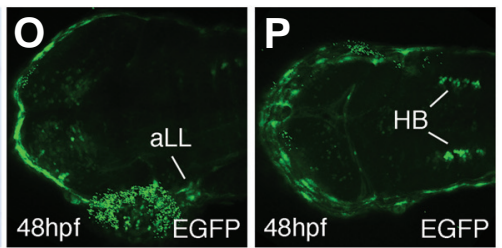
midbrain, and hindbrain (Fig. 2A). It is also expressed in the neurons that run the length of the spinal column (Fig. 2A). A dorsal view of a transgenic embryo at $24 \mathrm{hpf}$ also shows expression in the posterior lateral line ganglia (Fig. 2B). In the kidney, EGFP protein is present in the most distal region of the pronephric tubule and duct (Fig. 2 A,B, arrowhead). The expression domains of egfp mRNA coincide with those seen at the protein level. Expression is seen in the forebrain, midbrain, and hindbrain as well as in the neurons within the spinal cord (Fig. 2C). Strong expression is found within the posterior lateral line ganglia while kidney expression is limited to the distal tubule and duct (Fig. 2 C,D, arrowhead).

Endogenous / $h \times 1 a$ is found in the forebrain, specifically in the telencephalon and diencephaIon, as well as in the midbrain and hindbrain (Fig. 2E, bracket marks hindbrain). It is also seen in the spinal cord neurons (Fig. 2E). Outside of the CNS, expression of $/ h \times 1 a$ is restricted to the most proximal region of the pronephric tubule and to the tailbud (Fig. 2 E,F, arrow). Neither of these expression domains are conserved in Tg(Ihx1a:EGFP)pt303 embryos at $24 \mathrm{hpf}$. Loss of proximal tubule expression (Fig. 2 C,D compared to $2 \mathrm{E}, \mathrm{F}$ ) is consistent with the loss of anterior IM expression seen as early as 10 somites (Fig. $1 \mathrm{~L}$ compared to $1 \mathrm{R}$, white arrows). In addition, missing transgene expression in the tailbud (Fig. 2 C,D compared to 2 E,F) may be due to defuse, reduced egfp expression in the most posterior region of the notochord at 10 somites (Fig. $1 \mathrm{~L}$ compared to $1 \mathrm{R}$, arrowheads). These data suggest that additional regulatory elements may be necessary to completely mimic the endogenous expression pattern of Ihx1a.

By $48 \mathrm{hpf}$, strong expression of $\mathrm{Tg}$ (Ihx1a:EGFPpt303 at both the protein and RNA level is seen in the CNS (Fig. $2 \mathrm{G}, \mathrm{H}$ ). Expression is maintained in the posterior lateral line ganglia and is now apparent in the anterior lateral line ganglia (Fig. $2 \mathrm{G}, \mathrm{H}$ ). Expression is also seen in the otic vesicle, which is a conserved expression domain in Xenopus laevis (Karavanov etal. 1996), but is difficult to detect at the mRNA level (Fig. 2 G,H). However, transgene expression is no longer readily apparent in the kidney (Fig. $2 \mathrm{H}$, arrow). This expression pattern recapitulates what is seen for endogenous $/ h \times 1 a$ at this stage (Fig. 2l). By $72 \mathrm{hpf}$, EGFP protein and egfp mRNA are still present in the brain (Fig. $2 \mathrm{~J}, \mathrm{~K}$ ) but only the protein remains in spinal column neurons (Fig. 2J). Since endogenous $/ h \times 1 a$ is no longer expressed in the neurons of the spinal column (Fig. 2L), this may represent perdurance of GFP protein in this tissue.

To analyze the expression of Tg(Ihx1a:EGFP)ot303 in finer detail, we performed two-photon confocal microscopy on $24 \mathrm{hpf}$ embryos and $48 \mathrm{hpf}$ larvae, focusing on the CNS. At $24 \mathrm{hpf}$, transgene expression can be seen in both hemispheres of the midbrain (Fig. 2M) and this strong midbrain expression remains at $48 \mathrm{hpf}$ (Fig. 2N). Sectioning deeper into the larva, expression in the anterior lateral line ganglia (Fig. 2O) and the hindbrain (Fig. $2 \mathrm{P}$ ) becomes apparent. This transgene expression coincides with Ihx1a expression previously shown in histological sections

(Toyama and Dawid 1997). Overall, these data suggest that the expression of $T g$ (Ihx1a:EGFP)t303 recapitulates that of the endogenous Ihx 1a gene with only a few exceptions, and support the usefulness of this transgene for monitoring Ihx $1 a$ during development in live embryos.

\section{Responsiveness of $\mathrm{Tg}(\operatorname{lh} \times 1 \mathrm{a}: \mathrm{EGFP})^{\mathrm{pt} 303}$ to modulation of Nodal signaling}

To test whether Tg(Ihx1a:EGFPyt303 is responsive to signaling pathways known to regulate endogenous Ihx1a expression, we over-expressed ndr1, one of the zebrafish ligands of the Nodal pathway. It was previously shown that injection of low doses of ndr1 resulted in ectopic expression of $/ h \times 1 a$ in shield-stage embryos (Rebagliati et al. 1998). As compared to controls, injection of $\mathrm{Tg}$ (Ihx 1a:EGFP)t303 embryos with 25pg ndr1 mRNA led to an expansion of both transgene and endogenous Ihx1a expression (Fig. 3). These data suggest that Tg(Ihx1a:EGFPpt303, similar to the endogenous Ihx1a gene, is responsive to over-expression of Nodal and is therefore regulated by this pathway.

In a complimentary experiment, we utilized a small molecule to inhibit the Nodal pathway. SB-431542 blocks the activity of the type I activin receptor-like kinases (ALK) ALK4, ALK5, and ALK7 in mammalian cell culture by preventing phosphorylation of downstream effector molecules Smad2 and Smad3 (Inman etal. 2002). This compound inhibits Nodal signaling in zebrafish embryos (Hagos and Dougan 2007), and at high doses phenocopies sqt; cycdouble mutants, which eliminate early Nodal signals in the fish (Feldman et al. 1998; Hagos and Dougan 2007). As compared to controls, treatment of $\mathrm{Tg}$ (Ihx1a:EGFP)t303 embryos with $800 \mu \mathrm{M}$ SB-431542 led to a complete loss of transgene and endogenous /hx1a expression (Fig. 4 A-F). Eve1, a marker of the ventral side of the embryo, served as a negative control to show that treatment with SB-431542 does not disrupt all endogenous signaling events in the early embryo. As compared to controls, eve1 expression is still present in SB-431542 treated embryos (Fig. 4 G-O). Taken together, these data suggest that the effect 
seen with SB-431542 is specific, and that $\operatorname{Tg}$ (Ihx1a:EGFP)t303, like endogenous $/ h \times 1 a$, is regulated by Nodal signaling and is capable of responding to perturbations in this pathway.

\section{Discussion}

Here we present evidence that the transgenic line, Tg(Ihx1a:EGFP)t303, recapitulates the endogenous expression of $/ h \times 1 a$ and is regulated in the same manner during early development in the zebrafish embryo. Strong expression of this transgene begins during gastrula stages. In addition, Tg(Ihx1a:EGFP)t303 is detected in the pronephric kidney during
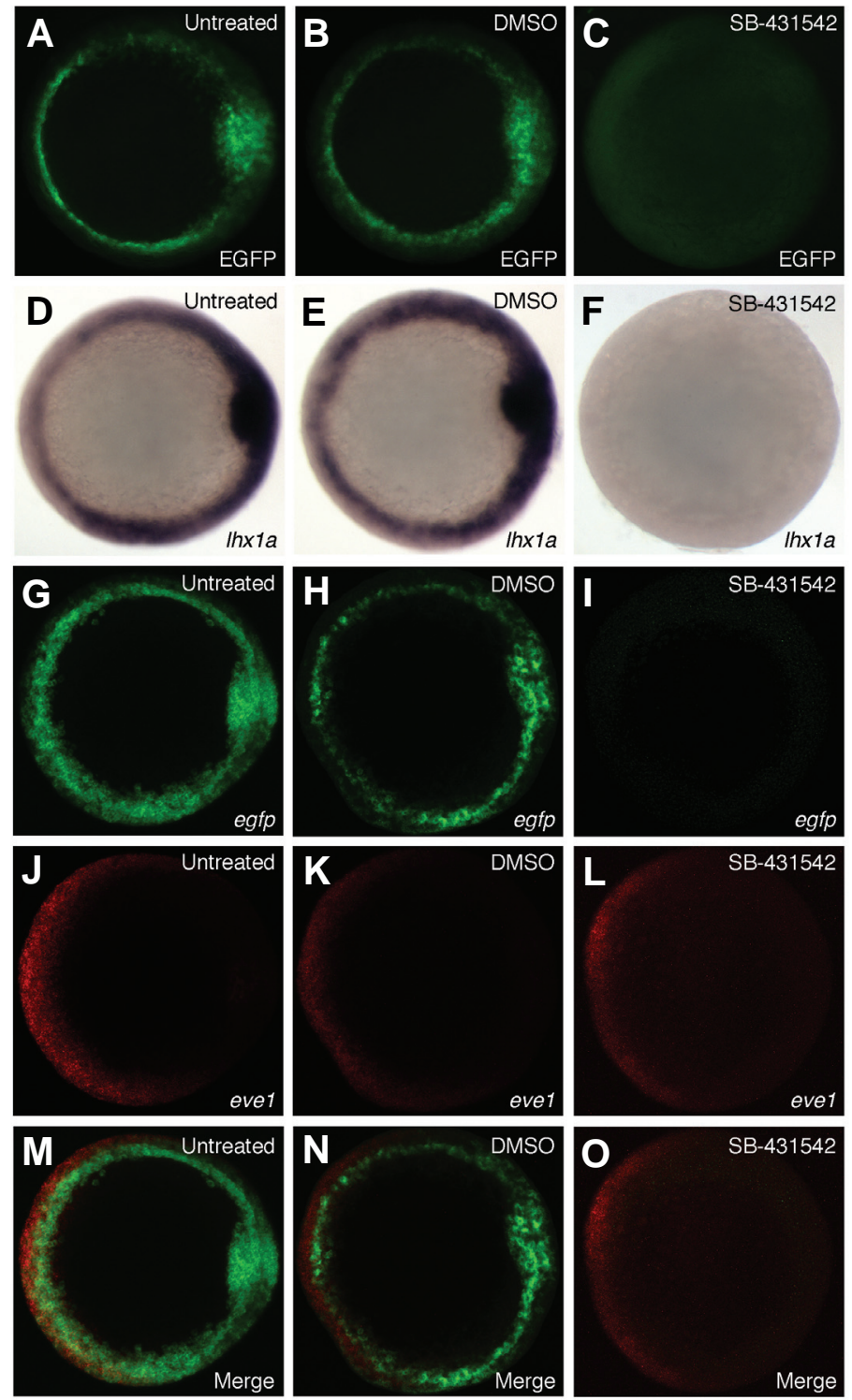

Fig. 4. Modulation of Tg(lhx1a:EGFP)pt303 expression by SB-431542. (A-O) Shield stage Tg(lhx1a:EGFP) ${ }^{\mathrm{pt} 303}$ embryos, animal view. (A,D,G,J,M) Untreated control embryos. (B,E,H,K,N) Embryos treated with $0.8 \%$ DMSO. (C,F,I,L,O) Embryos treated with $800 \mu \mathrm{M}$ SB-431542. (A-C) $\mathrm{Tg}\left(\mathrm{Ih} \times 1 \mathrm{a}\right.$ :EGFP) ${ }^{\mathrm{pt} 303}$ expression. (D-F) In situ hybridization for Ihx1a. (GO) Fluorescent in situ hybridization. (G-I) egfp. (J-L) eve1. (M-O) Merged images. early somitogenesis, which to date is the earliest published expression of a zebrafish transgenic reporter in the nephric field. Comparison with previous data suggests that this nephric expression of $\mathrm{Tg}$ (Ihx1a:EGFP) 303 may serve as a marker of kidney progenitor cells (Serluca and Fishman 2001). There are some inconsistencies between the transgene and the endogenous expression pattern of Ihx $1 \mathrm{a}$. Specifically, proximal tubule expression in the kidney and tailbud expression in the axial mesoderm are absent while diencephalon expression is aberrantly strong in the transgene. It is likely that regulatory regions are absent from this transgene, and this results in the discrepancies that we see between transgene and endogenous expression.

Our data demonstrate that $\mathrm{Tg}(\mathrm{Ih} \times 1 \mathrm{a}$ :EGFP)t303 responds to alterations in the Nodal pathway, suggesting that it is regulated in a manner similar to the endogenous gene. Lhx 1 a plays a number of important roles throughout development, and this transgene will serve as a useful tool not only for gaining insight into gene regulation but also for trying to understand some of the earliest steps of cell-fate specification. Finally, since zebrafish are wellsuited for small molecule screening (Vogt et al. 2009), this transgene could be used to screen for compounds that affect early gastrulation or kidney progenitor cells.

\section{Materials and Methods}

\section{Zebrafish husbandry}

Embryos expressing the $T g(I h x 1 a: E G F P)$ transgene were obtained from incrossing homozygous adults. Embryos were maintained at $28.5^{\circ} \mathrm{C}$ and were staged according to Kimmel et al. (Kimmel et al. 1995).

\section{Tg(Ihx1a:EGFP) construct design}

An $8.8 \mathrm{~kb}$ genomic region of the Ihx1a locus was obtained from BAC $184 \mathrm{f} 16$ by HindlII digest and sub-cloned into pBluescript II KS(+). The upstream $5.9 \mathrm{~kb} \mathrm{BamHI}$ fragment was removed from this vector to facilitate the introduction of an Ascl site, at base pair 12 downstream of the initiation codon, into the remaining $2.9 \mathrm{~kb}$ fragment. This allowed for the insertion of EGFP in-frame with the first three amino acids of Lhx1a. Sequencing confirmed that no spurious mutations were created by sitedirected mutagenesis. EGFP was amplified from the pEGFP-1 vector using primers with Mlul sites at their $5^{\prime}$ ends, an enzyme compatible with Ascl. Following insertion of EGFP into the construct, the $5.9 \mathrm{~kb} \mathrm{BamHI}$ fragment was returned to generate the original $8.8 \mathrm{~kb}$ genomic fragment in pBluescript II KS(+). The entire Ihx1a:EGFPfragment was then cloned into the pl-Scel vector by HindlII digest. 25pg of Ihx1a:EGFP/pl-Scel plasmid DNA was injected into 1-cell stage embryos along with the I-Scel restriction enzyme (NEB) (Thermes et al. 2002). These injected embryos were raised to adulthood and screened for EGFP expression in known Ihx1a expression domains. Three independent transgenic lines were isolated; Tg(Ihx1a:EGFP)pt301, Tg(Ihx1a:EGFP)pt302, and TgIIhx1a:EGFP)t303. All three lines displayed expression in the same domains, with slight variability in overall expression levels.

\section{In situ hybridization}

Embryos were fixed in 4\% paraformaldehyde (PFA) and processed for whole mount in situhybridization as described (Toyama and Dawid 1997) or for fluorescent in situ hybridization (FISH) (Schoenebeck et al. 2007), with the following modifications. Embryos were incubated in Cy3-tyramide or fluorescein-tyramide solution for one hour. RNA probes for Ihx1a, egfp, and eve 1 were used for this study.

\section{Zebrafish mRNA microinjection}

$m C h e r r y / p C S 2{ }^{+}$was linearized with Sacll and $n d r 1 / \mathrm{pCS} 2^{+}$was linear- 
ized with Notl. The mMessage mMachine SP6 kit (Ambion) was used to transcribe capped RNA for both genes as per manufacture's instructions. Tg(Ihx1a:EGFP)t303 embryos were injected with either 125pg mCherry mRNA or $100 \mathrm{pg}$ mCherry and $25 \mathrm{pg}$ ndr1 mRNA at the 1-cell stage. All embryos that expressed $m$ Cherryat shield stage were either fixed in $4 \%$ PFA or were analyzed for transgene expression.

\section{Chemical treatment of transgenic embryos}

SB-431542 (4- [4-(1,3 benzodioxol-5-yl)-5-(2-pyridinyl-1 H-imidazol2 -yl]benzamide) (Sigma) was stored as a $100 \mathrm{mM}$ stock in DMSO at $20^{\circ} \mathrm{C}$. Tg(Ihx1a:EGFP)t303 embryos at equivalent stages were arrayed into 12-well plates (maximum of 25/well) and were treated with $1.5 \mathrm{~mL}$ of E3, $0.8 \%$ DMSO or $1.5 \mathrm{~mL}$ of E3, $0.8 \%$ DMSO, $800 \mu \mathrm{M} \mathrm{SB}-431542$ between the 256 and 512-cell stage (Hagos and Dougan 2007). Drug or vehicle was removed at shield stage by washing five times with E3 media, and embryos were either fixed in 4\% PFA or were analyzed for transgene expression.

\section{Zebrafish Imaging}

Tg(Ihx1a:EGFP)ot303 embryos (shield through $24 \mathrm{hpf}$ ) were held in place on glass bottom culture dishes (MatTek Corporation) with $1 \%$ low melting point agarose. Larvae (48 and $72 \mathrm{hpf}$ ) were immobilized with tricaine and placed on similar dishes. Tg(Ihx1a:EGFP)t303 expression was imaged with either a Zeiss 510 Meta or a Leica TCS SP 5 confocal microscope. Z-stacks were taken for each image and projections were made using Image J. For Tg(Ihx1a:EGFP)t303 embryos treated with SB431542 or injected with ndr1 and/or mCherry RNA, images were taken with a Leica M205 FA epifluorescent microscope. Images were further analyzed with Adobe Photoshop CS3.

For optical sectioning, $T g$ (Ihx1a:EGFP)t303 embryos and larvae (24hpf and $48 \mathrm{hpf}$, respectively) were fixed in $4 \%$ PFA for 1 hour at room temperature. They were then positioned in a depression slide containing $3 \%$ methylcellulose and imaged with an Olympus Fluoview FV1000 MPE system. Images were analyzed in the same manner as above.

\section{Acknowledgements \\ We would like to thank Lance Davidson for help with confocal micros- copy; Dave Grainy and Gretchen Blasko for fish care; Wade Znosko for critical reading of the manuscript; David Kozlowski for identification of anatomical structures; Nathan Bahary for the mCherry expression plas- mid; Mike Rebagliati for the ndr1 expression plasmid and Michael Tsang for suggestions and advice. This work has been funded by the National Institutes of Health, USA:R01DK069403, R01HD053287, P30DK079307 and the NICHD Intramural Program.}

\section{References}

CARROLL, T.J. and VIZE, P.D. (1999). Synergism between Pax-8 and lim-1 in embryonic kidney development. Dev Bio/214: 46-59.

DAWID, I.B., BREEN, J.J. and TOYAMA, R. (1998). LIM domains: multiple roles as adapters and functional modifiers in protein interactions. Trends Genet 14: 156162.

FELDMAN, B., GATES, M.A., EGAN, E.S., DOUGAN, S.T., RENNEBECK, G., SIROTKIN, H.I., SCHIER, A.F. and TALBOT, W.S. (1998). Zebrafish organizer development and germ-layer formation require nodal-related signals. Nature
395: 181-185

HAGOS, E.G. and DOUGAN, S.T. (2007). Time-dependent patterning of the mesoderm and endoderm by Nodal signals in zebrafish. BMC Dev Bio/7: 22.

HUKRIEDE, N.A., TSANG, T.E., HABAS, R., KHOO, P.L., STEINER, K., WEEKS D.L., TAM, P.P. and DAWID, I.B. (2003). Conserved requirement of Lim1 function for cell movements during gastrulation. Dev Ce//4: 83-94.

INMAN, G.J., NICOLAS, F.J., CALLAHAN, J.F., HARLING, J.D., GASTER, L.M., REITH, A.D., LAPING, N.J. and HILL, C.S. (2002). SB-431542 is a potent and specific inhibitor of transforming growth factor-beta superfamily type I activin receptor-like kinase (ALK) receptors ALK4, ALK5, and ALK7. Mol Pharmacol 62: $65-74$.

KARAVANOV, A.A., SAINT-JEANNET, J.P., KARAVANOVA, I., TAIRA, M. and DAWID, I.B. (1996). The LIM homeodomain protein Lim-1 is widely expressed in neural, neural crest and mesoderm derivatives in vertebrate development. Int J Dev Bio/40: 453-461.

KIMMEL, C.B., BALLARD, W.W., KIMMEL, S.R., ULLMANN, B. and SCHILLING, T.F. (1995). Stages of embryonic development of the zebrafish. Dev Dyn203: 253-310.

KODJABACHIAN, L., KARAVANOV, A.A., HIKASA, H., HUKRIEDE, N.A., AOKI, T., TAIRA, M. and DAWID, I.B. (2001). A study of Xlim1 function in the Spemann-Mangold organizer. Int J Dev Bio/45: 209-218.

REBAGLIATI, M.R., TOYAMA, R., FRICKE, C., HAFFTER, P. and DAWID, I.B. (1998). Zebrafish nodal-related genes are implicated in axial patterning and establishing left-right asymmetry. Dev Bio/199: 261-272.

REBBERT, M.L. and DAWID, I.B. (1997). Transcriptional regulation of the Xlim-1 gene by activin is mediated by an element in intron I. Proc Nat/ Acad Sci USA 94: 9717-9722.

SCHIER, A.F. and TALBOT, W.S. (2005). Molecular genetics of axis formation in zebrafish. Annu Rev Genet 39: 561-613.

SCHOENEBECK, J.J., KEEGAN, B.R. and YELON, D. (2007). Vessel and blood specification override cardiac potential in anterior mesoderm. Dev Ce//13: 254 267.

SERLUCA, F.C. and FISHMAN, M.C. (2001). Pre-pattern in the pronephric kidney field of zebrafish. Development 128: 2233-2241.

SHAWLOT, W. and BEHRINGER, R.R. (1995). Requirement for Lim1 in headorganizer function. Nature 374: 425-430.

TAIRA, M., JAMRICH, M., GOOD, P.J. and DAWID, I.B. (1992). The LIM domaincontaining homeo box gene Xlim-1 is expressed specifically in the organizer region of Xenopus gastrula embryos. Genes Dev6: 356-366.

THERMES, V., GRABHER, C., RISTORATORE, F., BOURRAT, F., CHOULIKA, A. WITTBRODT, J. and JOLY, J.S. (2002). I-Scel meganuclease mediates highly efficient transgenesis in fish. Mech Dev118: 91-98.

TOYAMA, R. and DAWID, I.B. (1997). lim6, a novel LIM homeobox gene in the zebrafish: comparison of its expression pattern with lim1. Dev Dyn 209: 406417.

TOYAMA, R., O'CONNELL, M.L., WRIGHT, C.V., KUEHN, M.R. and DAWID, I.B (1995). Nodal induces ectopic goosecoid and lim1 expression and axis duplication in zebrafish. Development 121: 383-391.

VOGT, A., CHOLEWINSKI, A., SHEN, X., NELSON, S.G., LAZO, J.S., TSANG, M and HUKRIEDE, N.A. (2009). Automated image-based phenotypic analysis in zebrafish embryos. Dev Dyn 238: 656-663.

WATANABE, M., REBBERT, M.L., ANDREAZZOLI, M., TAKAHASHI, N., TOYAMA, R., ZIMMERMAN, S., WHITMAN, M. and DAWID, I.B. (2002). Regulation of the Lim-1 gene is mediated through conserved FAST-1/FoxH1 sites in the first intron. Dev Dyn 225: 448-456. 


\section{Further Related Reading, published previously in the Int. J. Dev. Biol.}

See Special Issue Pattern Formation edited by Michael K. Richardson and Cheng-Ming Chuong at: http://www.ijdb.ehu.es/web/contents.php?vol=53\&issue=5-6

Comparison of Lim1 expression in embryos of frogs with different modes of reproduction Michael Venegas-Ferrín, Norihiro Sudou, Masanori Taira and Eugenia M. del Pino Int. J. Dev. Biol. (2010) 54: 195-202 (doi: 10.1387/ijdb.092870mv)

Expression dynamics of the LIM-homeobox genes, Lhx1 and Lhx9, in the diencephalon during chick development Xiangnan Sun, Hirotomo Saitsu, Kohei Shiota and Makoto Ishibashi Int. J. Dev. Biol. (2008) 52: 33-41

\section{A study of Xlim1 function in the Spemann-Mangold organizer}

Kodjabachian, L., Karavanov, A.A., Hikasa, H., Hukriede, N.A., Aoki, T., Taira, M. And Dawid, I.B. Int J Dev Biol (2001) 45: 209-218.

Defects of the body plan of mutant embryos lacking Lim1, Otx2 or Hnf3beta activity S J Kinder, T E Tsang, S L Ang, R R Behringer and P P Tam Int. J. Dev. Biol. (2001) 45: 347-355

Role of the anterior visceral endoderm in restricting posterior signals in the mouse embryo A Perea-Gomez, M Rhinn and S L Ang Int. J. Dev. Biol. (2001) 45: 311-320

The LIM homeodomain protein Lim-1 is widely expressed in neural, neural crest and mesoderm derivatives in vertebrate development

Karavanov, A.A., Saint-Jeannet, J.P., Karavanova, I., Taira, M. And Dawid, I.B.

Int J Dev Biol (1996) 40: 453-461.

Expression of mesoderm markers in Xenopus laevis Keller explants J P Saint-Jeannet, A A Karavanov and I B Dawid

Int. J. Dev. Biol. (1994) 38: 605-611

5 yr ISI Impact Factor $(2008)=3.271$
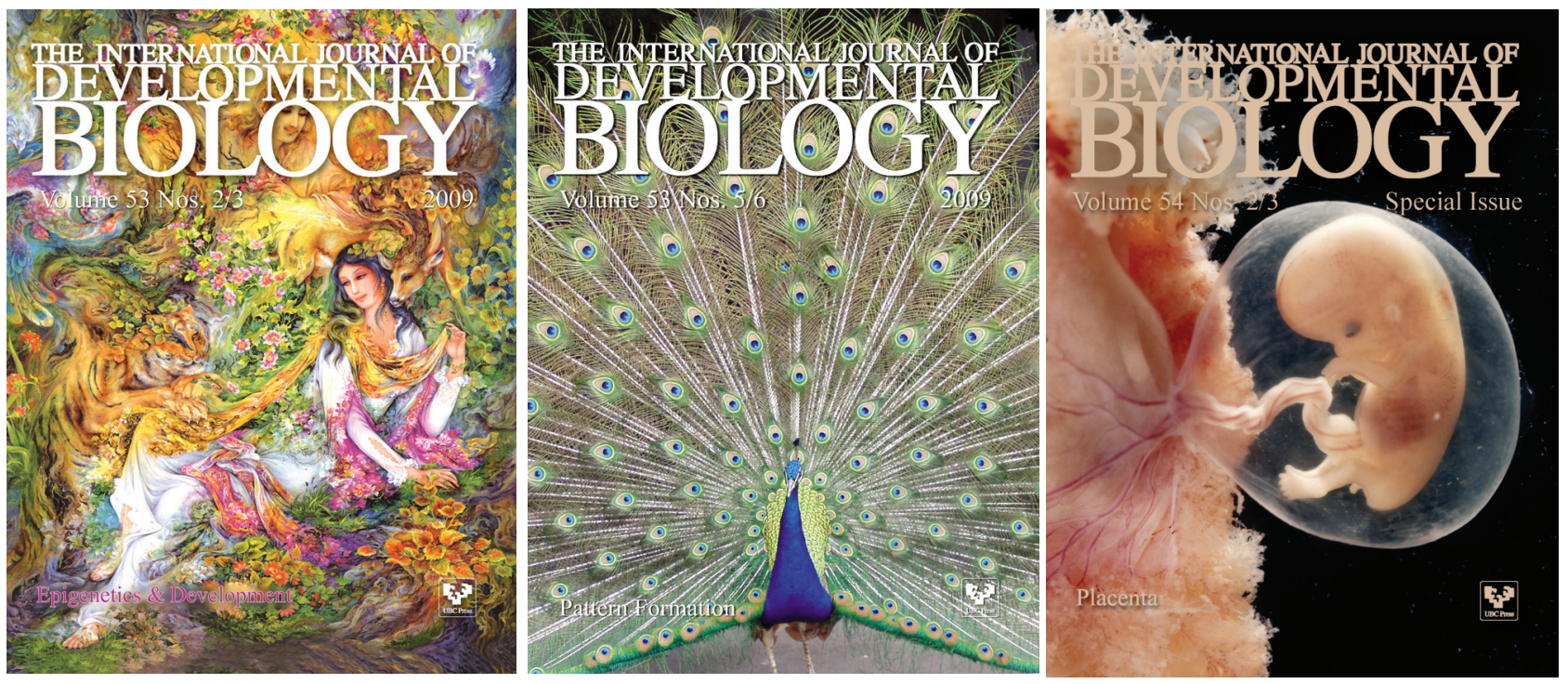